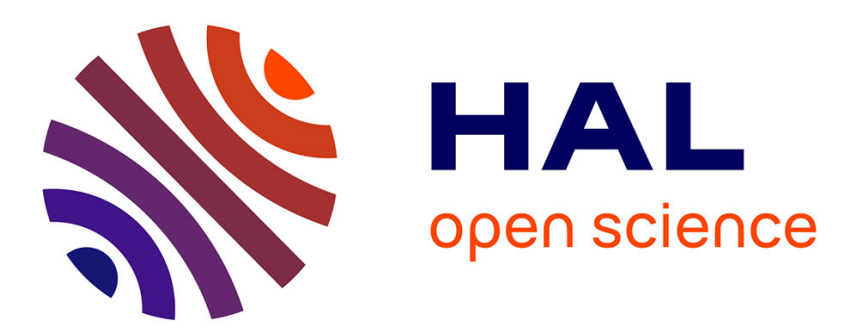

\title{
Installed jet noise source analysis by microphone array processing
}

\author{
Renaud Davy, Frédéric Mortain, Maxime Huet, Thomas Le Garrec
}

\section{To cite this version:}

Renaud Davy, Frédéric Mortain, Maxime Huet, Thomas Le Garrec. Installed jet noise source analysis by microphone array processing. 25th AIAA/CEAS Aeroacoustics Conference, May 2019, DELFT,

Netherlands. hal-02262210

\section{HAL Id: hal-02262210 \\ https://hal.science/hal-02262210}

Submitted on 2 Aug 2019

HAL is a multi-disciplinary open access archive for the deposit and dissemination of scientific research documents, whether they are published or not. The documents may come from teaching and research institutions in France or abroad, or from public or private research centers.
L'archive ouverte pluridisciplinaire HAL, est destinée au dépôt et à la diffusion de documents scientifiques de niveau recherche, publiés ou non, émanant des établissements d'enseignement et de recherche français ou étrangers, des laboratoires publics ou privés. 


\title{
Installed jet noise source analysis by microphone array processing
}

\author{
Renaud Davy ${ }^{1}$, Frédéric Mortain ${ }^{2}$, Maxime Huet ${ }^{2}$, and Thomas Le Garrec ${ }^{2}$ \\ DAAA, ONERA, Université Paris Saclay \\ F-92322Châtillon - France
}

This paper presents the identification of jet noise sources carried out at ONERA in the framework of the European Project JERONIMO. A dedicated test campaign was conducted in the anechoic wind tunnel CEPRA19 for a UHBR dual-stream nozzle with and without wing model installed. Arrays of microphone were implemented in order to apply advanced processing for noise source identification. The analysis focused on the wing installation effects. Correlated monopoles model was used in order to identify the different noise sources radiation occurring in installed jet configurations. The results projected in far field have been assessed with the farfield measurement. The acoustic localization processing applied to the numerical simulation demonstrates a good agreement with the experiments. Tonal jet-flap interaction noise is also highlighted, showing strong correlation between source at bypass exit and flap trailing edge.

\section{Introduction}

$\mathrm{T}$ he increase in air traffic volumes and the continuous growing of environmental constraints led the air transport industry to developed new engine concept. One of the solutions, addressed in the FP7 European project JERONIMO, is to improve ducted turbofan engine. As far as cycle efficiency has been optimized by Engine Manufacturers in the last decade, limited additional benefits could be expected, and the efforts are now focused on propulsive efficiency improvement. This way leads to study Ultra High Bypass Ratio (UHBR) engine. The installation constraints of the engine conduct to increasingly close-coupled architectures. As a consequence, even more strong jet installation noise, induced by the jet mixing interaction with the wing and the flap is expected, especially at take-off and landing; the first due to maximum jet thrust, the second connected to the high lift devices spreading.

This problematic has been studied both experimentally and numerically in the JERONIMO project. This paper deals with the experimental part realized in the ONERA's Cepra19 anechoic opened test-section wind tunnel. The identification of installed jet noise has been studied using microphone arrays measurements. Different signal processing techniques based on uncorrelated or correlated noise sources models ${ }^{1,2}$ are compared in this paper. The source identification processing has also been applied on numerical simulation signals ${ }^{3}$ for a cross analysis of both approach. The test setup is briefly presented in Sec. II. The main issues of the methodology are given in Sec. III. Finally the installation effects are analysed in Sec. IV.

\section{Test setup}

The CEPRA19 wind tunnel is operated with the two meters convergent nozzle equipped with the jet simulator mock-up. The model used is the JERONIMO Ultra High Bypass Ratio (BPR 15) axisymmetric nozzle. Isolated configurations were firstly tested. Then, installation effects of powerplant have been studied by mounting a 3D high-lift airfoil without pylon. To perform acoustic measurements under flight path, the wing is mounted vertically. (see Figure 1).

\footnotetext{
${ }^{1}$ Research scientist, Aerodynamics, aeroelasticity and acoustics department, renaud.davy@onera.fr

${ }^{2}$ Research scientist, Aerodynamics, aeroelasticity and acoustics department
} 


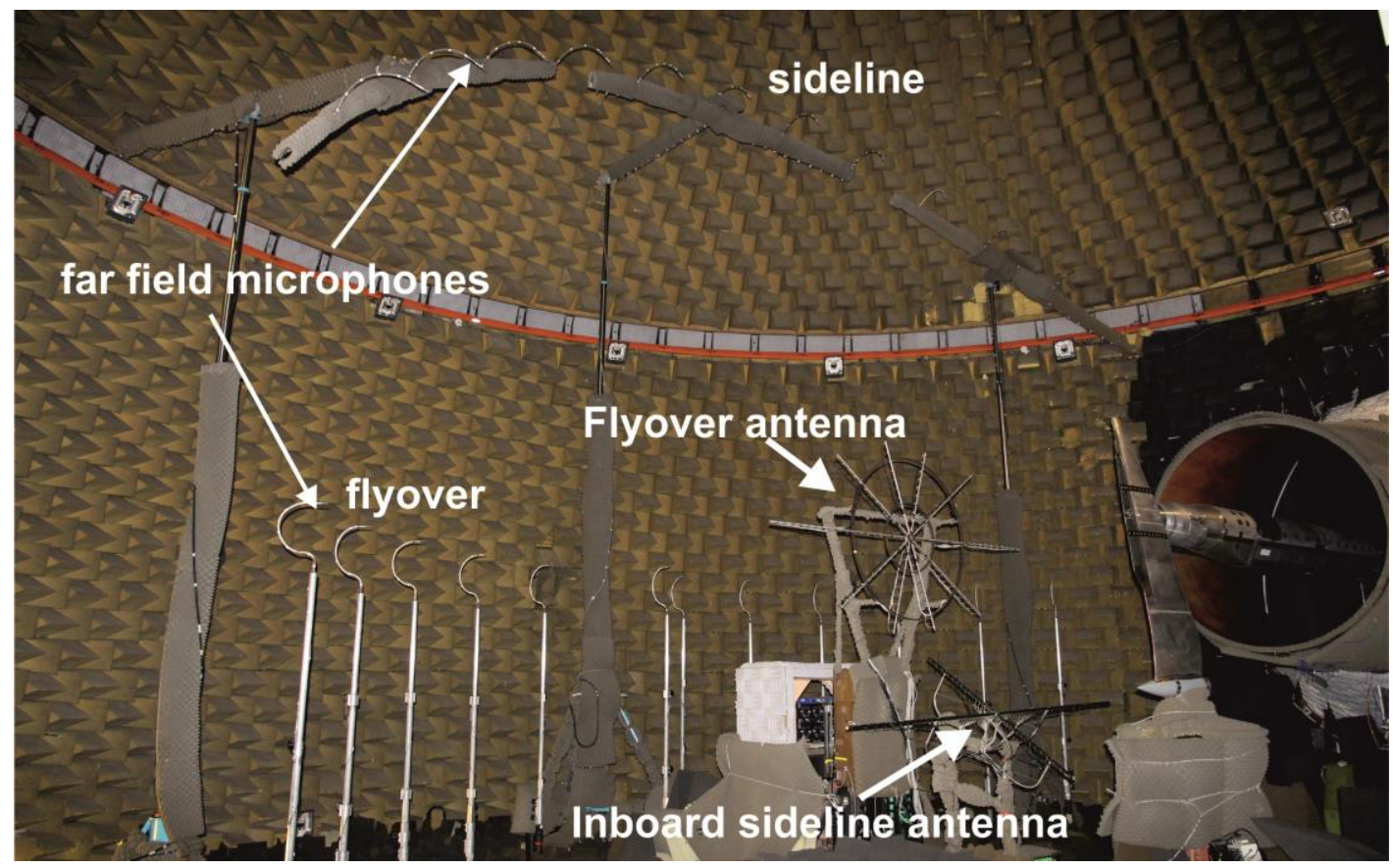

Fig. 1: JERONIMO test in CEPRA19

Different effects have been investigated for several jet operating conditions. As jet installation effect for close-coupled installation is of major concern, the influence of the flap deflection angle and the relative position of the jet with respect to the wing, have also been investigated.

The far field directivities are measured in the flyover plane ( 0 deg. in the azimuthal arc) and sideline plane (56 deg. in the azimuthal arc) with two 6 meter-radius arrays centered on the secondary exhaust center of the nozzle. Each array is composed of 12 microphones located every $10^{\circ}$ between $40^{\circ}$ upstream to $150^{\circ}$ downstream with respect to the nozzle axis at inlet. For noise sources identification, two 2D microphone arrays instrumented with 1/4" pressure field microphones were implemented for the test. The first one was set in the $\mathrm{x}-\mathrm{z}$ plane at $1.75 \mathrm{~m}$ from the jet axis in the flyover direction (see Fig. 2). It is composed of 131 microphones distributed on 5 arms. The second one is a cross-shaped array fixed in the inboard sideline direction $\left(-56^{\circ}\right.$ in the azimuthal arc) also at $1.75 \mathrm{~m}$ from the jet axis. The $\mathrm{X}$ arm of $2.85 \mathrm{~m}$ includes 71 microphones, the second arm is composed of 35 microphones distributed along $1.5 \mathrm{~m}$. In this paper, only results related to the flyover direction are presented.

All the microphone signals were synchronously acquired on a B\&K LanXI data acquisition system at the sampling frequency of $262144 \mathrm{~Hz}$.

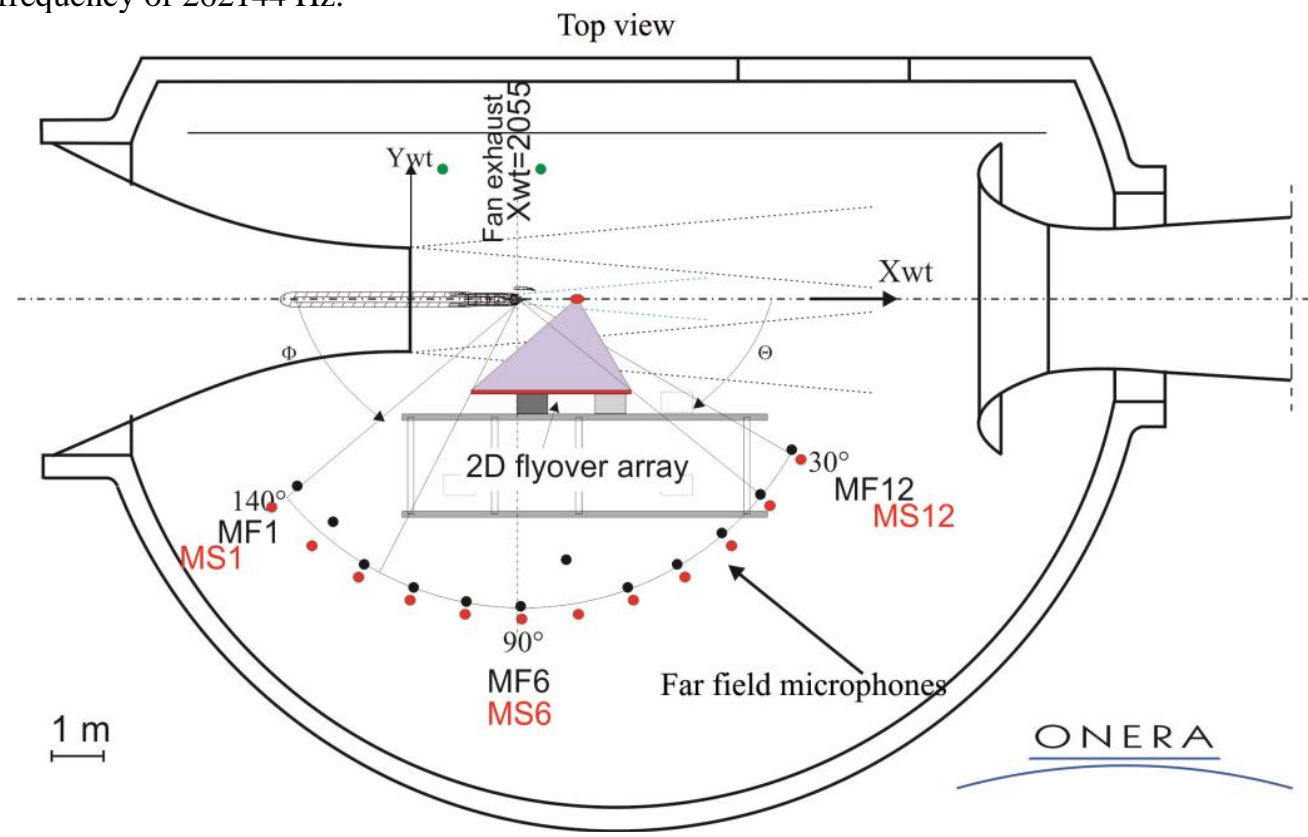

Fig. 2: Scheme of the JERONIMO test setup in CEPRA19 


\section{Noise source identification technique}

\section{A. Deconvolution algorithms}

The array processing methods used by ONERA in JERONIMO are derived from the well-known DAMAS and DAMAS-C microphone array techniques see Ref. [1,2], and has been more detailed in previous papers $^{4,5}$. Whatever the methodology is, the task consists in finding the optimal representation of noise sources reproducing the measurements, in particular the microphone cross-spectral matrix, as closely as possible. The first one considers the noise sources as a distribution of uncorrelated monopoles. It goes back to determine the diagonal of the source cross spectra matrix. To reproduce the directive acoustic pattern radiated by the jet, an advanced microphone array technique based on correlated source terms could be preferably applied. The methodology addressed by the DAMAS-C algorithm consists in restoring cross-spectra between source points.

Jet noise is modelled by a set of $N_{S}$ monopoles at prescribed positions. The task is to determine source parameters $P$, for instance the amplitudes of the monopoles or their cross-spectra, that optimally reproduce the microphone array measurements, specifically the microphone Cross-Spectral Matrix (CSM) $\Gamma$ :

$$
P=\arg \min \left\|\Gamma-\Gamma^{\bmod e l}(P)\right\|
$$

Equation 1

where $\Gamma^{\text {model }}$ is a model CSM. The acoustic pressure $p_{m}$ generated from a set of $N_{S}$ monopoles at the position of the $\mathrm{m}^{\text {th }}$ microphone is given by

$$
p_{m}=\sum_{i=1}^{N S} \alpha_{i} G_{i, m}
$$

Equation 2

where $\alpha_{i}$ is the amplitude of the $\mathrm{i}^{\text {th }}$ monopole and $G_{i, m}$ is the Green's function between $\mathrm{i}$ and $\mathrm{m}$. Equation 2 is defined for a given frequency $\mathrm{f}$. Thus, for correlated monopoles, the model CSM is

$$
\Gamma_{m, n}^{\text {model }}(C)=\overline{p_{m} p_{n}^{*}}=\Sigma_{i, j} G_{i, m} C_{i, j} G_{j, n}{ }^{*}
$$

Equation 3

where $C_{i, j}=\overline{\alpha_{i} \alpha_{j}{ }^{*}}$ is the source CSM between the $\mathrm{i}^{\text {th }}$ source and $\mathrm{j}^{\text {th }}$ source. Similarly, for uncorrelated monopoles $\left(C_{i, j}=0\right.$ for $\left.i \neq j\right)$,

$$
\Gamma_{m, n}^{\text {model }}(C)=\sum_{i} G_{i, m} G_{i, n}^{*} S_{i}
$$

Equation 4

where $S_{i}=C_{i, i}$ are the source auto-powers (for the frequency f)

Due to the number of variables to restore, $N_{S}^{2}$, the problem defined by Equation 1 is ill-posed. In order to control the deconvolution process, basic physical properties concerning the sought solution $C$ are then introduced. For instance, jet noise source cross-spectra $C\left(\vec{x}_{i}, \vec{x}_{j}\right)$ should vanish when the distance between $\vec{x}_{i}$ and $\vec{x}_{j}$ is larger than several jet diameters. Furthermore, by ensuring that the scanned source domain is sufficiently large to encompass all the jet noise sources $C\left(\vec{x}_{i}, \vec{x}_{j}\right)$ should vanish at the boundaries of the source domain. Following the Tikhonov's approach, these two conditions are introduced by turning the initial optimization Equation 1 into

$$
C=\arg \min \left\|\Gamma-\Gamma^{\bmod e l}(C)\right\|^{2}+\lambda^{2}\|W \circ C\|^{2}
$$

Equation 5

where $\lambda$ is a regularization parameter, $W$ is an appropriate weighting function, and o is the term-to-term product.

In the usual approach of uncorrelated noise sources, the localization function coherence $\gamma^{2}=\frac{G^{+} \Gamma G}{\|G\|^{2} \operatorname{Tr}(\Gamma)}$ is

known to be a good operator for the localization of noise sources ${ }^{6}$. An extension to correlated noise sources was proposed by Fleury ${ }^{5}$. This operator is used to define the weighting function W. The regularization parameter $\lambda$ is optimized from the L-curve method $^{7}$. 
Computations are made with different values of these parameters over a limited number of frequencies and iterations. For each value, the convergence is checked: the norms of the residue and the regularized solution are used according to the L-curve criterion (log-log plot of the residual norm versus the solution norm). The optimal regularization parameter is considered to be located at the corner of the L-curve, which is expected to provide the best trade-off between the residual norm and the solution norm.

\section{B. Acoustic extrapolation and assessment of the source cross-spectra}

By using the deconvoluted source cross-spectral matrix $C(f)$, the acoustic level $\Gamma(\vec{x}, \vec{x}, f)$ generated in $\vec{x}$ at frequency $f$ in an environment of known Green's function $G_{i}^{e x t r a}(\vec{x}, f)$, between $\vec{x}$ and source points $i \in S_{\text {restricted }}(f)$ over a restricted area along the jet axis (to be defined in the remainder), is provided by

$$
\Gamma^{e x t r a}(\vec{x}, \vec{x}, f)=\sum_{i, j \in S_{\text {restriced }}(f)}^{N_{S}} G_{i}^{\text {extra }}(\vec{x}, f) C_{i, j}(f) G_{j}^{\text {extra, }}(\vec{x}, f)
$$

Equation 5

Equation 5 is used to assess the deconvoluted source cross-spectra by comparison of the extrapolated acoustic levels $\Gamma^{\text {extra }}(\vec{x}, \vec{x}, f)$ to the position of the far-field microphones of Cepra19 and actual independent measurements in these positions. For the extrapolation, the ad hoc Green's function of Cepra19 $G_{i}^{\text {extra }}(\vec{x}, f)$ is used, namely either the free-field Green's function in quiescent medium, if there is no flight flow, or the sophisticated Green's function incorporating both acoustic convection effects and refraction effects in presence of jet-surrounding flight flow (deviation of acoustic rays and amplitude modification). Furthermore, the atmospheric attenuation of acoustic waves is also introduced into the far-field extrapolation of the jet noise source terms. The integration domain in Equation 5 could be reduced to a domain $i \in S_{\text {restricted }}(f)$ in order to avoid aliased sources in the high-frequency range.

We also have to take into account diffraction effect caused by the microphone mounting system. In a first step this correction is basically introduced considering the assumption of diffraction by an infinite isolated cylinder and is applied a posteriori considering the same effect for all microphones of the antenna. Work is ongoing to better take into account this effect and/or reduce it with new mounting arrangement. Nevertheless results presented below (Fig. 7) demonstrate that the spectral shape is much better reproduced when this correction is taken into account.

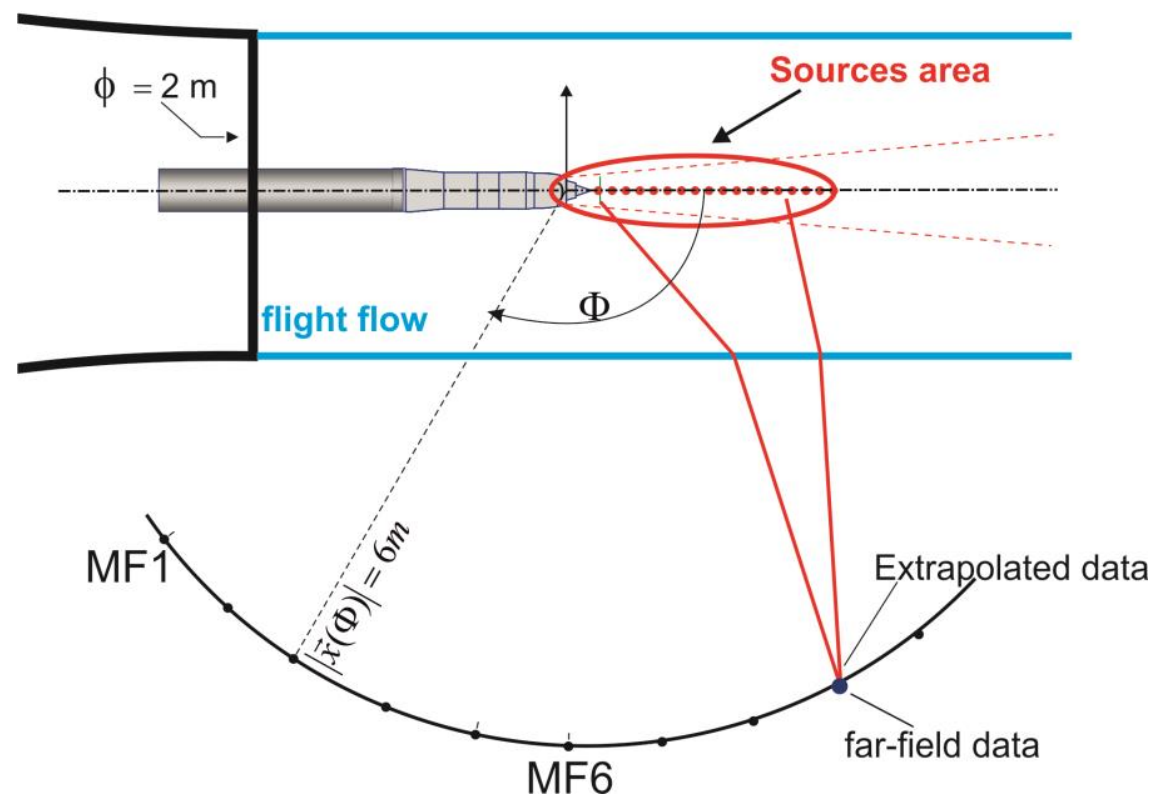

Fig. 3: Scheme of the acoustic extrapolation principle 


\section{Numerical simulations approach}

For one jet operating conditions (called OP10) of the experimental test matrix, numerical simulation have been performed both in isolated and installed configurations. This work has been already presented in a previous AIAA paper ${ }^{3}$, where the numerical simulation conditions and results are detailed. The purpose here is to apply the acoustics localization techniques on these results and compare them with the experimental data.

The simulations are performed with the elsA software ${ }^{8}$ developed at ONERA which solves the compressible Navier-Stokes equations on structured multiblock meshes.

The physical modelling used for this study is the Zonal Detached Eddy Simulation (ZDES) ${ }^{9}$, which is a hybrid RANS/LES approach aiming at treating the attached boundary layers in RANS mode and the free shear layers in LES mode. For the isolated cases, far-field pressure time signals are reconstructed from the unsteady aerodynamic flow fields stored on surfaces surrounding the jet in the near field, using the Ffowcs-Williams and Hawkings integral formulation ${ }^{10}$ with additional flux terms proposed by Rahier et al. ${ }^{11}$. For the far-field noise computation of the installed configuration, a CFD/CAA coupling approach is used (NRI approach ${ }^{12}$ ).

In addition to the simulation /experiments comparison, we take profit of the simulation to investigate the influence of the antenna position with respect to the nozzle exit. The time pressure signals of the simulation have been extracted at the equivalent microphone position of the flyover antenna mounted during the experimental test campaign and for a virtual position shifted $1.5 \mathrm{~m}$ downstream in order to be more in the maximum lobe directivity of the mixing noise. Table 1 summarizes the conditions of the computed configurations and whose of the experimental cases presented in this paper.

The duration of the simulation available for the array processing is $74 \mathrm{~ms}$. The cross-spectral matrices of the virtual microphones signals are computed in doing only 4 averages. The resulting frequency resolution is respectively $64 \mathrm{~Hz}$ and $54 \mathrm{~Hz}$ for the isolated and installed simulations. Then the same array data processing is applied as for the experimental data (see below).

\begin{tabular}{|c|c|c|c|c|c|c|}
\hline \multicolumn{7}{|c|}{ Experiment } \\
\hline $\begin{array}{c}\text { Jet operating } \\
\text { name }\end{array}$ & $\begin{array}{c}\mathrm{Pt} / \mathrm{Pamb} \\
\text { (Core) }\end{array}$ & $\begin{array}{l}\text { Pt/Pamb } \\
\text { (Bypass) }\end{array}$ & $\begin{array}{l}\mathrm{Tt}(\mathrm{K}) \\
\text { (Core) }\end{array}$ & $\begin{array}{c}\mathrm{Tt}(\mathrm{K}) \\
\text { (Bypass) }\end{array}$ & $\mathrm{U} \infty$ & $\begin{array}{l}\text { Antenna } \\
\text { position }\end{array}$ \\
\hline OP3-S & 1.691 & 1.681 & 329.3 & 328.2 & 18.6 & $\mathrm{X}_{\mathrm{s}}+0.65 \mathrm{~m}$ \\
\hline OP3-F2b & 1.691 & 1.681 & 330.6 & 326.9 & 61. & $\mathrm{X}_{\mathrm{s}}+0.65 \mathrm{~m}$ \\
\hline OP10-S & 1.221 & 1.333 & 764.1 & 322.0 & 13.7 & $\mathrm{X}_{\mathrm{s}}+0.65 \mathrm{~m}$ \\
\hline OP10-F4 & 1.224 & 1.335 & 762.8 & 318.0 & 90.1 & $\mathrm{X}_{\mathrm{s}}+0.65 \mathrm{~m}$ \\
\hline \multicolumn{7}{|c|}{$\begin{array}{r}\text { Simulation } \\
\end{array}$} \\
\hline & $\begin{array}{c}\mathrm{Pt} / \mathrm{Pamb} \\
\text { (Core) }\end{array}$ & $\begin{array}{l}\text { Pt/Pamb } \\
\text { (Bypass) }\end{array}$ & $\begin{array}{l}\mathrm{Tt}(\mathrm{K}) \\
\text { (Core) }\end{array}$ & $\begin{array}{c}\mathrm{Tt}(\mathrm{K}) \\
\text { (Bypass) }\end{array}$ & 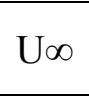 & \\
\hline OP10-F4 & 1.222 & 1.342 & 764.0 & 325.0 & 90 & $\begin{array}{c}\mathrm{X}_{\mathrm{s}}+0.65 \mathrm{~m} \\
\mathrm{X}_{\mathrm{s}}+2.2 \mathrm{~m}\end{array}$ \\
\hline
\end{tabular}

Table 1: Operating conditions of the presented configurations

\section{Data analysis}

This chapter presents the analysis of identification noise sources based on array processing methods. The computations are obviously much more time consuming when correlated monopoles are considered. Consequently the number of monopoles must be limited. For both processing, the following parameters were used:

- 2D DAMAS: $101 \times 31=3131$ uncorrelated monopoles distributed in the vertical plane passing though the jet axis $(Y=0): \quad X=1.85 \mathrm{~m}$ up to $X=4.85 \mathrm{~m}$ by step of $3 \mathrm{~cm}$ (101 points),

$\mathrm{Z}=-0.45 \mathrm{~m}$ up to $\mathrm{Z}=0.45 \mathrm{~m}$ by step of $3 \mathrm{~cm}$ ( 31 points);

The regularization parameter is $\lambda=0.8$, and 20000 iterations were calculated

- 2D DAMAS-C: $22 \times 13=286$ correlated monopoles distributed in the vertical plane passing though the jet axis $(\mathrm{Y}=0)$ : $\mathrm{X}=1.9 \mathrm{~m}$ up to $\mathrm{X}=4.0 \mathrm{~m}$ by step of $10 \mathrm{~cm}$ ( 22 points),

$\mathrm{Z}=-0.3 \mathrm{~m}$ up to $\mathrm{Z}=0.3 \mathrm{~m}$ by step of $5 \mathrm{~cm}$ (13 points),

The regularization parameter is $\lambda=0.6$ and 500000 iterations were calculated

The deconvoluted acoustics maps were calculated in the frequency range from $256 \mathrm{~Hz}$ up to $20 \mathrm{kHz}$. Three parameters were investigated during the test campaign: jet operating conditions, flight effect and installation 
effect. The data analysis present below will focus on the installation effect. The influence of the processing parameters will be also exhibited, especially the comparison of uncorrelated and correlated monopoles models. The acoustic results of numerical simulation will be analyzed throughout array processing. The effect of antenna positions with respect to the nozzle on the identification noise sources directivities will be also investigated thanks to the numerical simulations.

\section{Influence of the sources model considered}

As jet noise and jet installation noise present strong directivity pattern, a sources model able to reproduce such acoustic field is required. That's why, signal processing technique based on DAMAS-C algorithm was developed at ONERA. In this paper, the data analysis will be based on this method only. Nevertheless, comparison of both models is firstly presented in this paragraph. Fig. 4 presents comparison of octave band maps calculated by both approaches. We can observe that in high frequencies the sources distribution is comparable, but in lower frequencies corresponding to the mixing noise, the noise sources region is more concentrated downstream with the correlated monopoles model. The maximum level is located at about five secondary exhaust diameter from the bypass exhaust. Fig. 5presents the comparison between the direct far field measurements (black lines) and the spectra extrapolated from the source cross-spectral matrices obtained by both techniques using uncorrelated (red lines) or correlated monopoles models (green lines). The results correspond to an isolated nozzle configuration. The spectra comparison is plotted at three emission angles. We can observe a quite good agreement, excepted in low frequency (below $800 \mathrm{~Hz}$ ) where an underestimation up to $10 \mathrm{~dB}$ is noticed. At low frequency the DAMAS-C results is not fully converged. Much more iterations were needed but the computation was stopped for time consumption reason. Nevertheless even if the low frequencies in the rear arc are still underestimated the results are much better with the correlated model. The directivity pattern given in Fig. 6 confirms that this model is able to reproduce directive source (at least up to now in the array angle aperture) in contrast with uncorrelated one.

In order to demonstrate the microphones mountings diffraction effect, the results of DAMAS-C is compared with and without taking into account this correction in Fig. 7. As mentioned earlier, we observed that the spectral shape is better reproduced when this correction is considered. Otherwise the high frequency range is overestimated

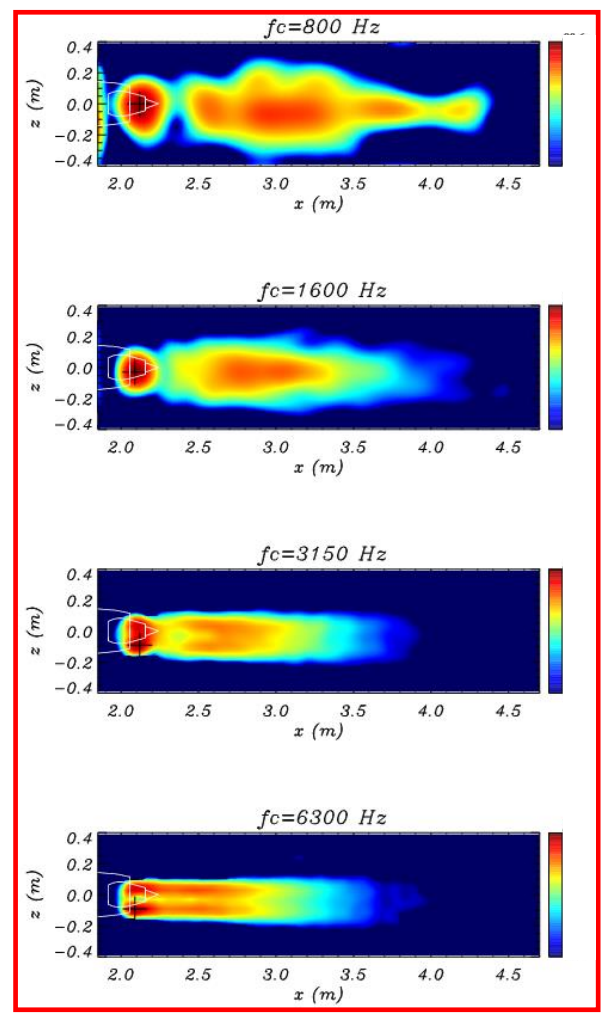

I10 - OP3-S
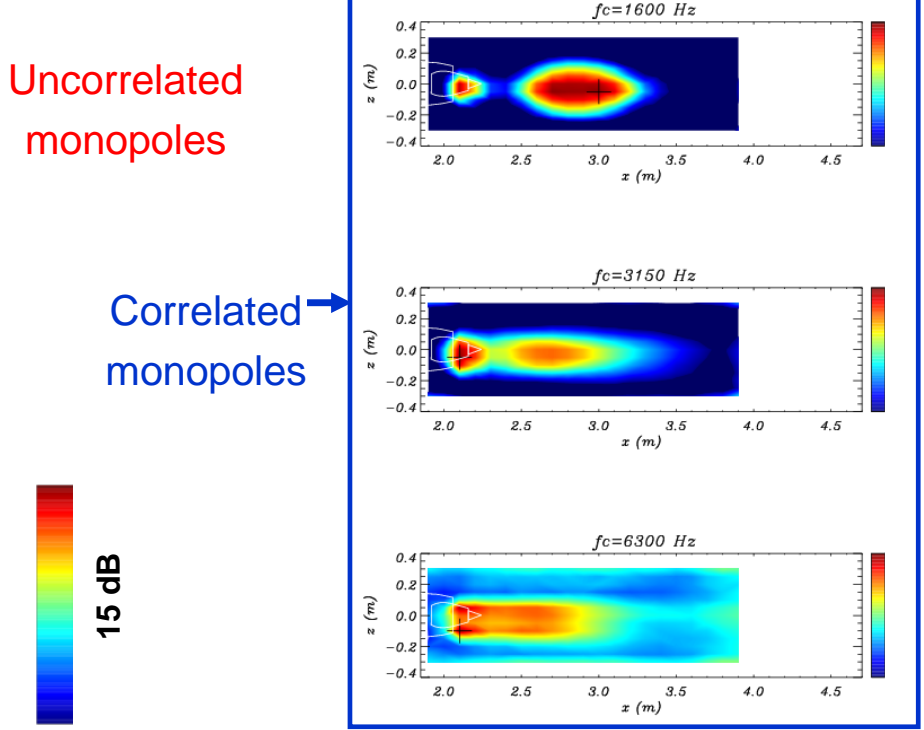

Fig. 4: Deconvoluted acoustic maps in octave band. Comparison between uncorrelated and correlated monopoles models. 

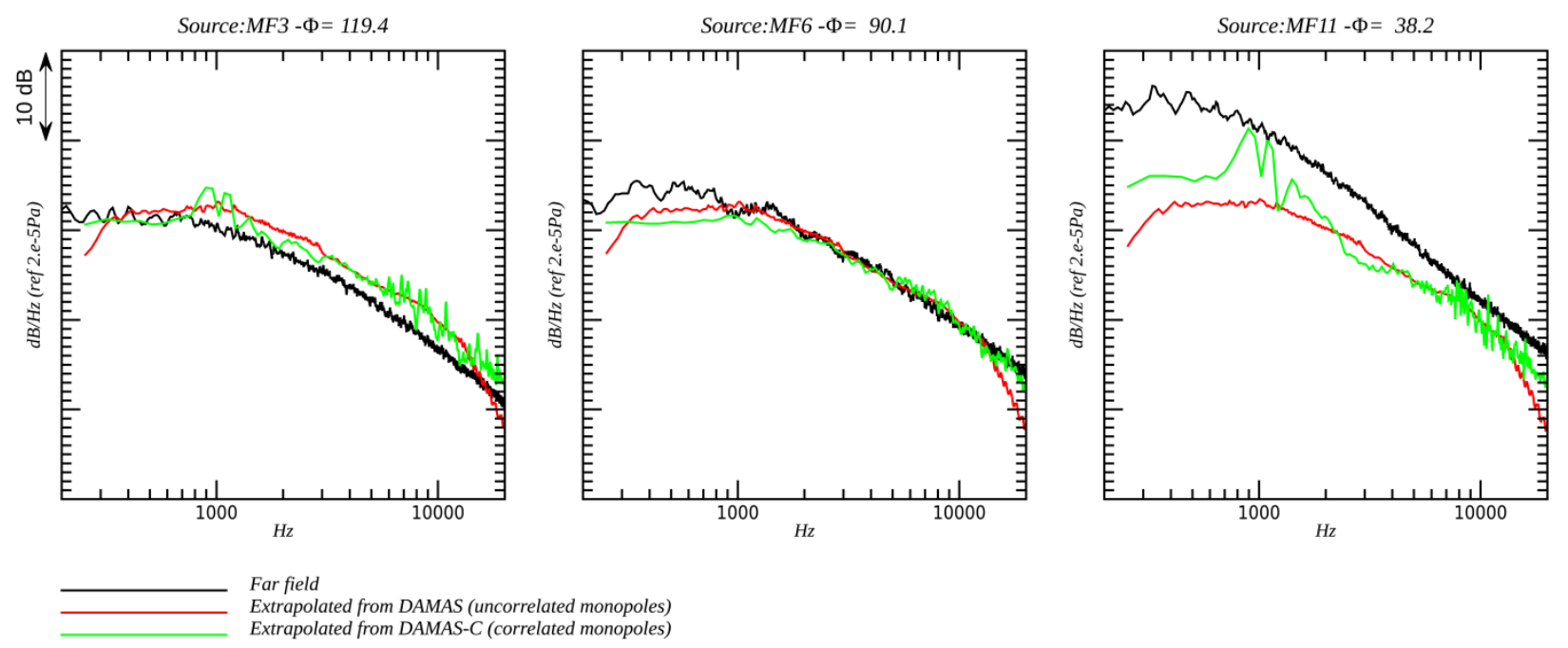

Fig. 5: Comparison of direct far field measurements with extrapolation from the cross spectra sources obtained with uncorrelated or correlated monopoles models - Isolated nozzle configuration (static caseOP3) - Flyover spectra

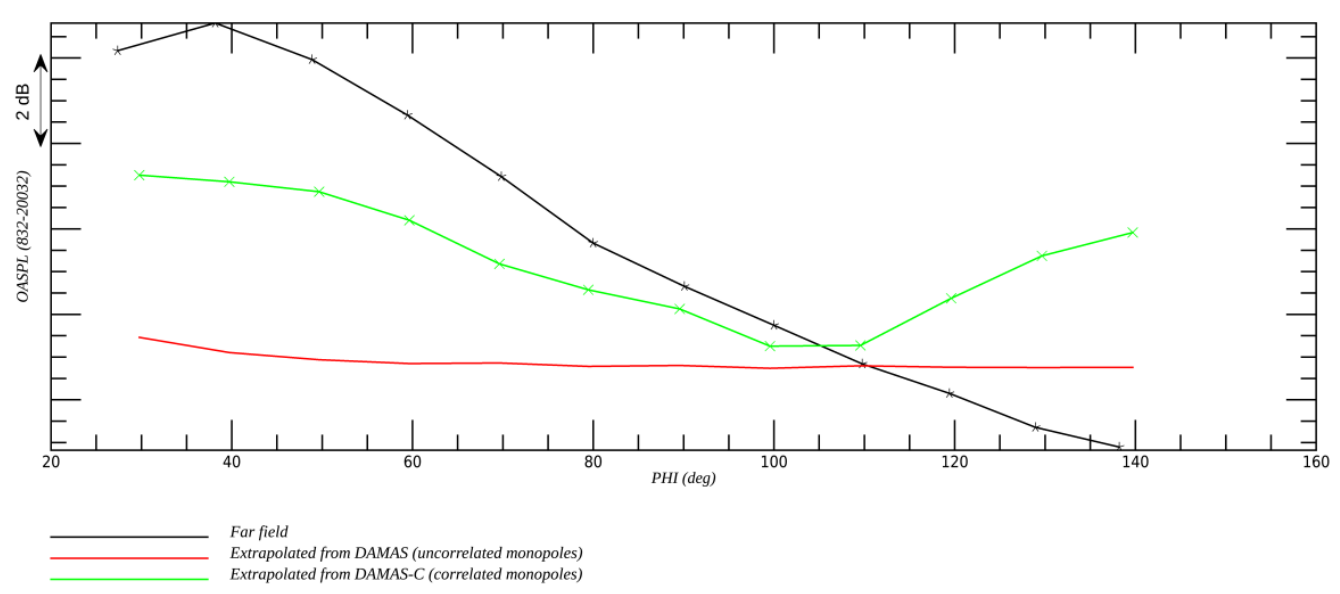

Fig. 6: Comparison of direct far field measurements with extrapolation from the cross spectra sources obtained with uncorrelated or correlated monopoles models - Isolated nozzle configuration (static caseOP3)- Flyover directivities
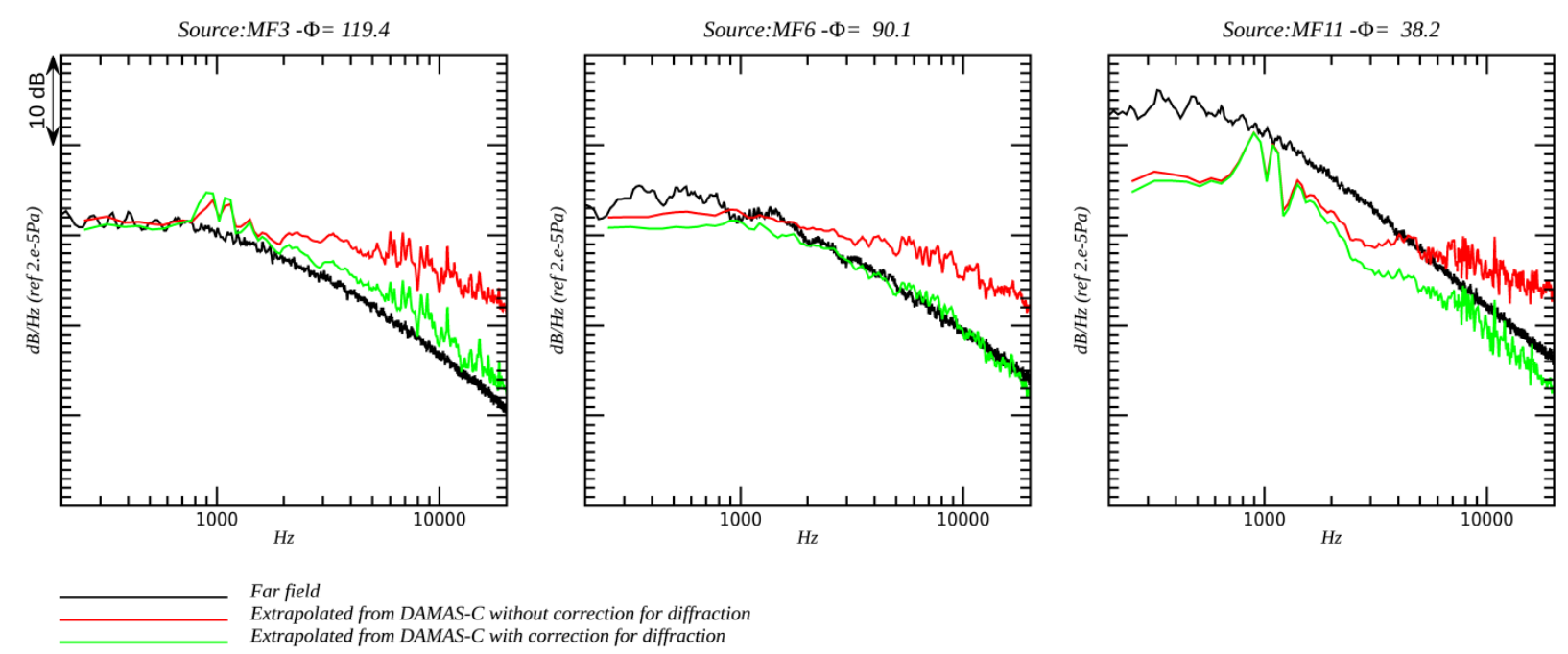

Fig. 7: Influence of correction for diffraction effect by the microphone support on the extrapolated data. 


\section{E. Installation effect}

One of the main objectives of these tests was to study the installation effect. Two parameters have been investigated, the flap deflection angle and the relative position of the jet with respect to the wing.

The Fig. 8 illustrates the installation effect for an approach configuration for five octave bands. It compares the acoustics map in isolated nozzle configuration and in installed configuration, without flight effect. The color scale is adjusted on the maximum level of each plot with the same dynamic range $(15 \mathrm{~dB})$

The strong jet installation noise which occurs in low frequency is well exhibited. For the octave band $500 \mathrm{~Hz}$, it masks the mixing noise and is also dominant up to octave band $2000 \mathrm{~Hz}$. Its contribution decreases for higher frequency. For the isolated configuration, if the mixing noise contribution is well localized in the octave band $500 \mathrm{~Hz}$, above the source are localized at nozzle exit. Two reasons could suggest that this source at nozzle exit is probably overestimated. Firstly, due to the large size of the nozzle some rig noise occurs for high bypass condition, secondly the position of the antenna implies that the mixing noise source located about $1.2 \mathrm{~m}$ downstream the nozzle exit, is mainly viewed upstream by the antenna which minimize its contribution.

This last hypothesis has been investigated thanks to the numerical simulations. The Fig. 9 compares the results of the sources identification processing applied for both positions of the array of microphones. The color scale is the same for all plots in order to compare here the absolute amplitude. It demonstrates that the jet noise source level determined is higher when the antenna is located more downstream. Improvements are required to better recover large directivity pattern: on the one hand on the source model, on the other hand, on the microphone array distribution.
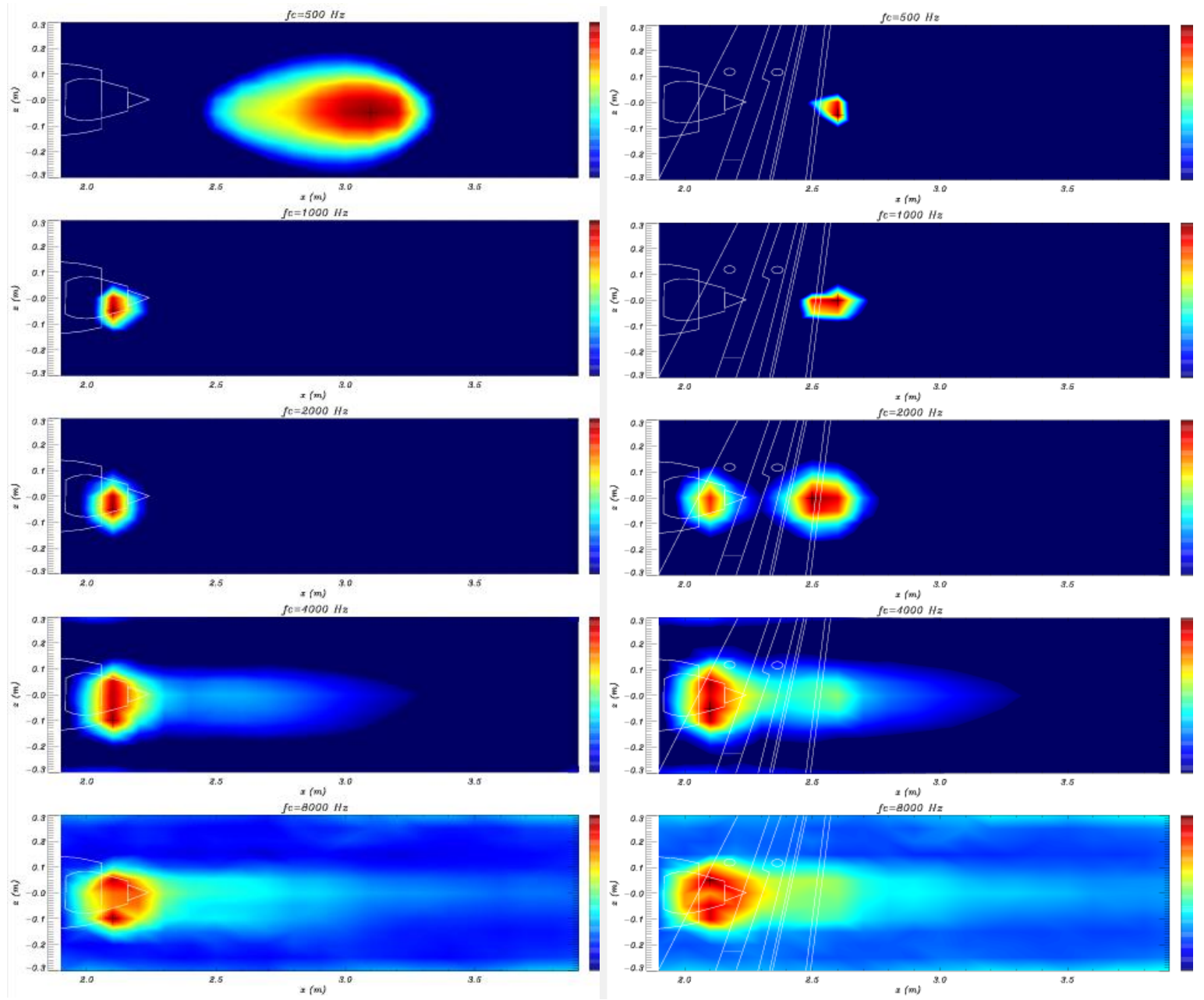

Fig. 8: Installation effect: OP10 static case - DAMAS-C- 2D Flyover antenna - Acoustic maps in octave band. - Left: isolated nozzle, right: wing installed configuration - (colorscale: 15 dB dynamic) 


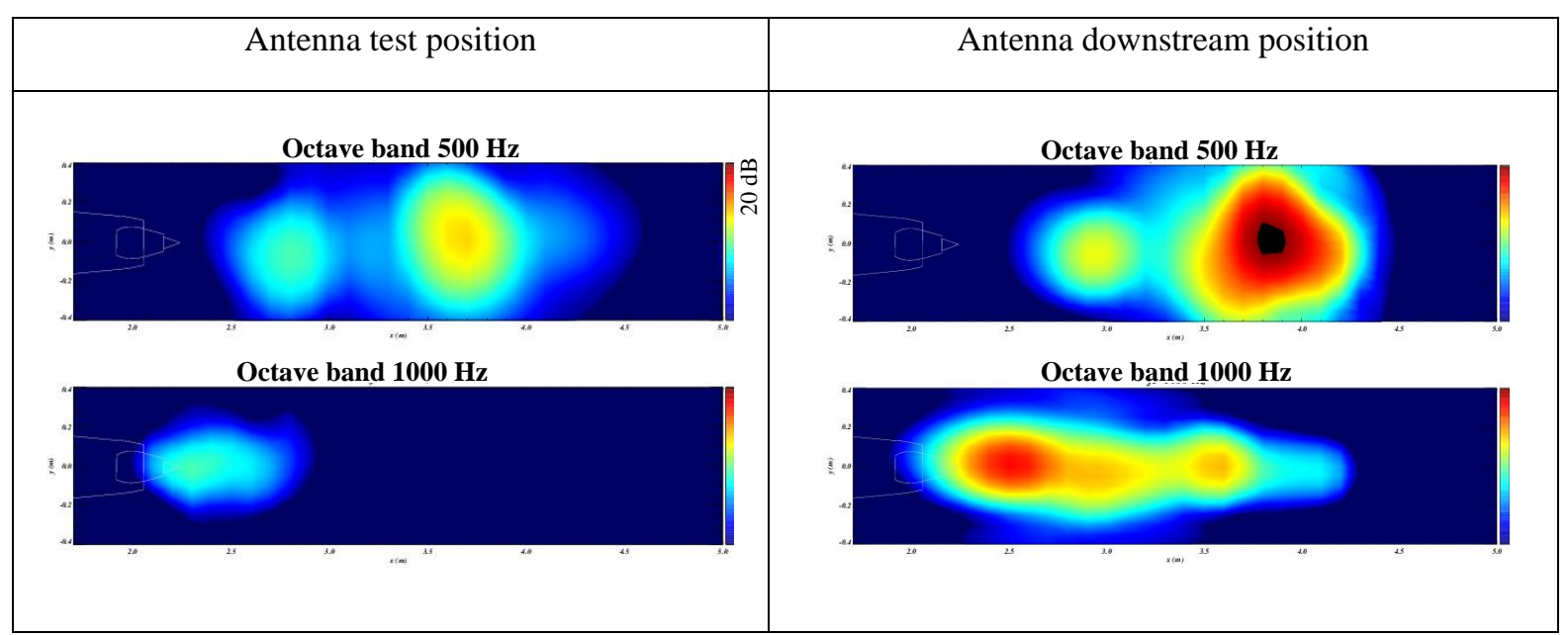

Fig. 9: Influence of the microphones array position on the acoustic maps (DAMAS-C) - Numerical simulation for isolated nozzle - OP10- Flight effect $(90 \mathrm{~m} / \mathrm{s})$-Left: Test position, right: downstream position - (common color scale: $20 \mathrm{~dB}$ dynamic)

\section{Comparison of noise sources identification between Experiment/Numerical simulation data}

This jet operating condition with flight effect has been computed in isolated and installed configurations. The Fig. 10 compares the noise sources identification processing applied on numerical simulation and on the experimental data for both configurations. The numerical simulation reproduces very well the installation effect. The jet/flap interaction noise is accurately localized at the flap trailing edge as for the experimental data. The main difference between the simulation and experiment is the stronger noise source obtained experimentally at the bypass exhaust. But in this configuration with high external flight velocity, the rig noise significantly contaminates the jet noise measurement in the experiment, which probably explains the difference with the simulation when the jet/flap interaction noise is not dominant. Apart from this difference, the numerical data exhibit the main sources at about $4.5 \mathrm{~m}$ for the octave band $500 \mathrm{~Hz}$, which is probably due to the vortex generated at flap tip intersecting the CAA sources injection surface. Up to now we cannot conclude if this source is purely a numerical artefact or a physical noise due to the interaction between the tip vortex and the jet shear layer. This source is not detected in the experiments which privilegiates the first assumption. But the source map should also be extended in the $\mathrm{x}+$ and $\mathrm{y}+$ directions in order to determine if the outboard tip vortex generates the same pattern. Indeed, the mean flow is not symmetrical in the y direction due to the presence of the wing.

The power spectral densities of the radiated pressure obtained by simulation are compared with the CEPRA19 farfield measurements in Fig. 11 and the directivities integrated between $400 \mathrm{~Hz}$ and $2 \mathrm{kHz}$ in Fig. 12. Again the farfield measurement for the isolated configuration must be considered carefully as they are strongly contaminated by the rig noise, but at least they give a reference background noise with regard to the installed configuration. That's why we will only notice that downstream, where the jet mixing noise is dominant, the four curves are almost superposed in low frequency suggesting that this contribution is not strongly affected by the installation effect and is similarly reproduced by both simulations. For the isolated simulation as mentioned in a previous paper $^{3}$ a spurious contribution affects the simulation above $3 \mathrm{kHz}$. This unexpected noise is attributed to an artefact of the computation coming from a vortex roll-ups at the initial stages of the fan mixing layer. Concerning the installed configuration the agreement with experiments is satisfying. Furthermore the directivities integrated in the range $[400-2000 \mathrm{~Hz}]$ present a good behavior compared to experiment, and the effect of jet/flap interaction versus the isolated jet is also comparable to the experimental one. Indeed the downstream directivity is reproduced for jet noise in isolated nozzle whereas upstream directivity stresses the jet/flap main contribution in installed configurations. 


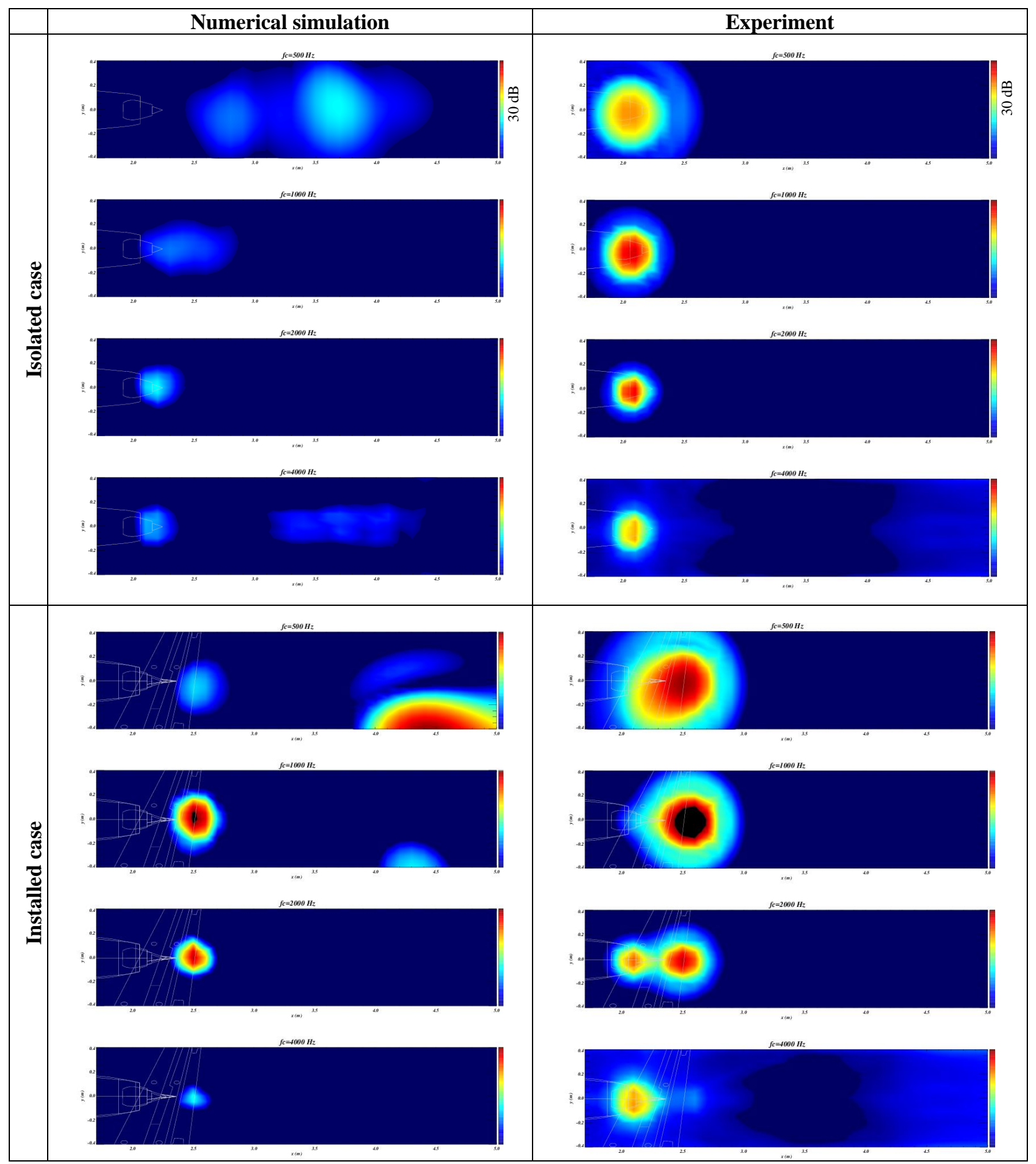

Fig. 10: Installation effect OP10- Flight effect $(90 \mathrm{~m} / \mathrm{s})$ - Left: numerical simulation, right: ExperimentsUpper: Isolate, lower: Installed - (common color scale: 30 dB dynamic) 

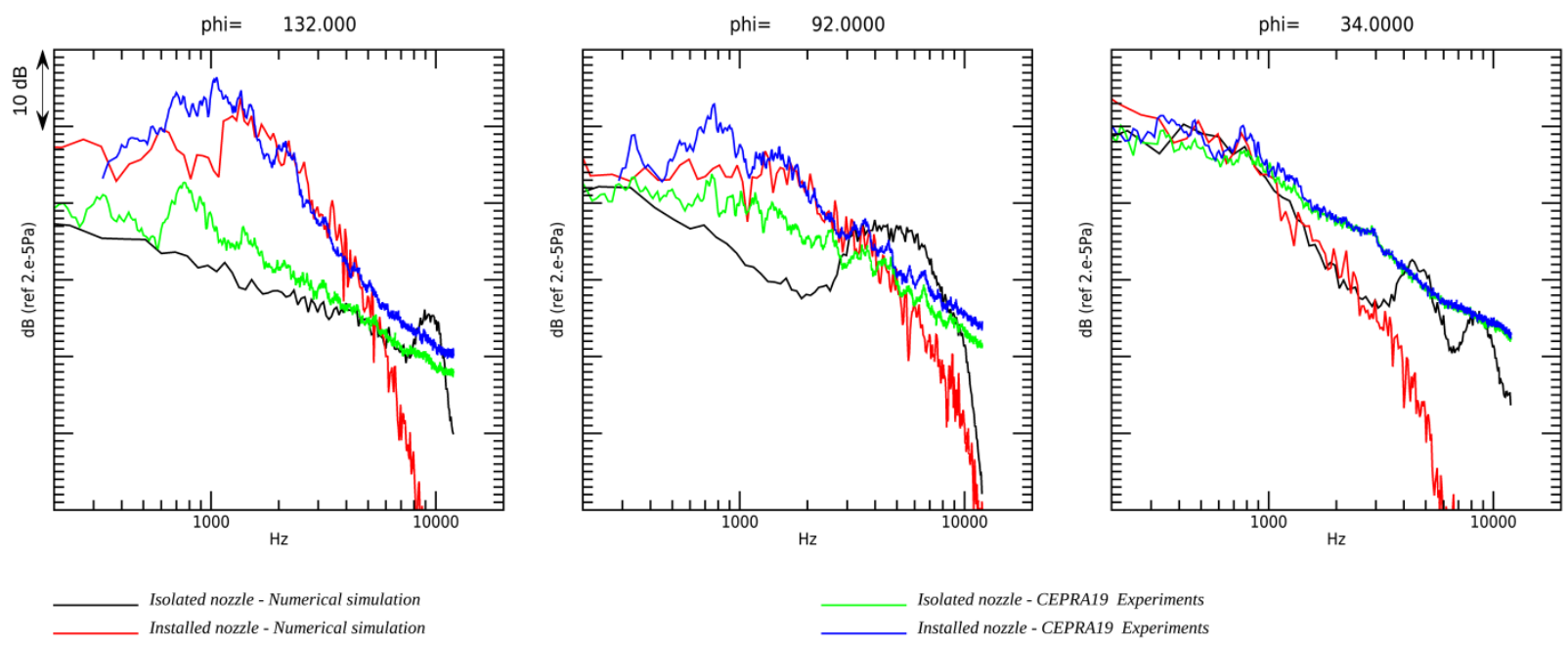

Fig. 11: Numerical simulation / CEPRA19 experiments farfield spectra comparison. Isolated/Installed nozzle: OP10- Flight effect $(90 \mathrm{~m} / \mathrm{s})$

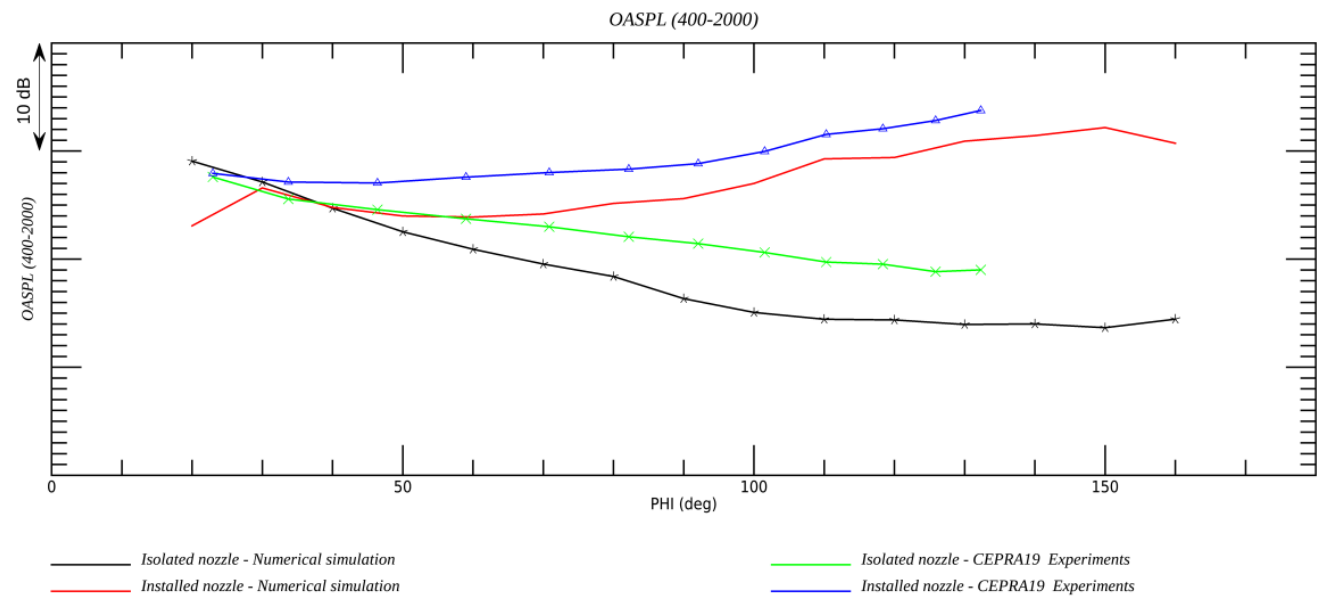

Fig. 12: Numerical simulation / CEPRA19 experiments farfield directivities comparison. Isolated/Installed nozzle: OP10- Flight effect $(90 \mathrm{~m} / \mathrm{s})$

\section{Assessment of the sources cross spectra decomposition}

In order to assess the acoustic source maps obtained with DAMASC process, the sources are propagated in farfield and compared with the direct farfield measurement following the methodology described in section B. This work is presented in Fig. 13. The noise sources extrapolation is done in separating three different areas: nozzle exit, jet/flap interaction area and jet mixing noise. The corresponding source spectra and the total noise are superimposed with the measured farfield spectra at three different angles. We can notice firstly a good agreement of the total reconstructed noise with the direct measurement. Secondly we clearly observe the dominant contribution of the jet-flap interaction up to about $2 \mathrm{kHz}$ whereas the nozzle exit noise becomes dominant at higher frequencies. The directivities are calculated for these two ranges of integration [400$2000 \mathrm{~Hz}$ ], and [2-20 kHz]. In the low frequency range, again we observe the good agreement with far field directivity dominated by the upstream radiation of the jet/flap interaction noise. But we also see a directivity pattern that peaks near $90^{\circ}$ for the nozzle exit noise which could be due to the contribution of the internal rig noise at these frequencies. 

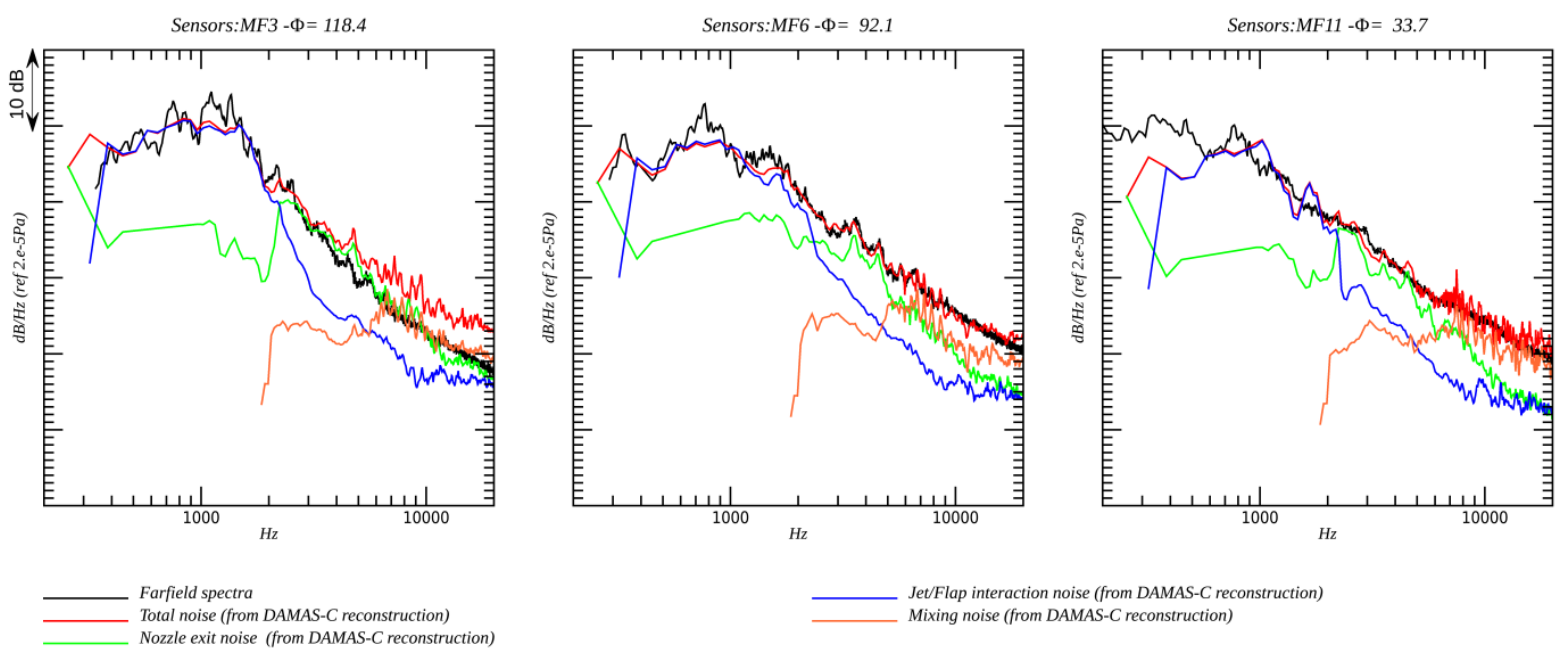

Fig. 13: Comparison of direct far field measurements with reconstructed source spectra from DAMAS-C sources identification. Installed nozzle: OP10- Flight effect $(90 \mathrm{~m} / \mathrm{s})$
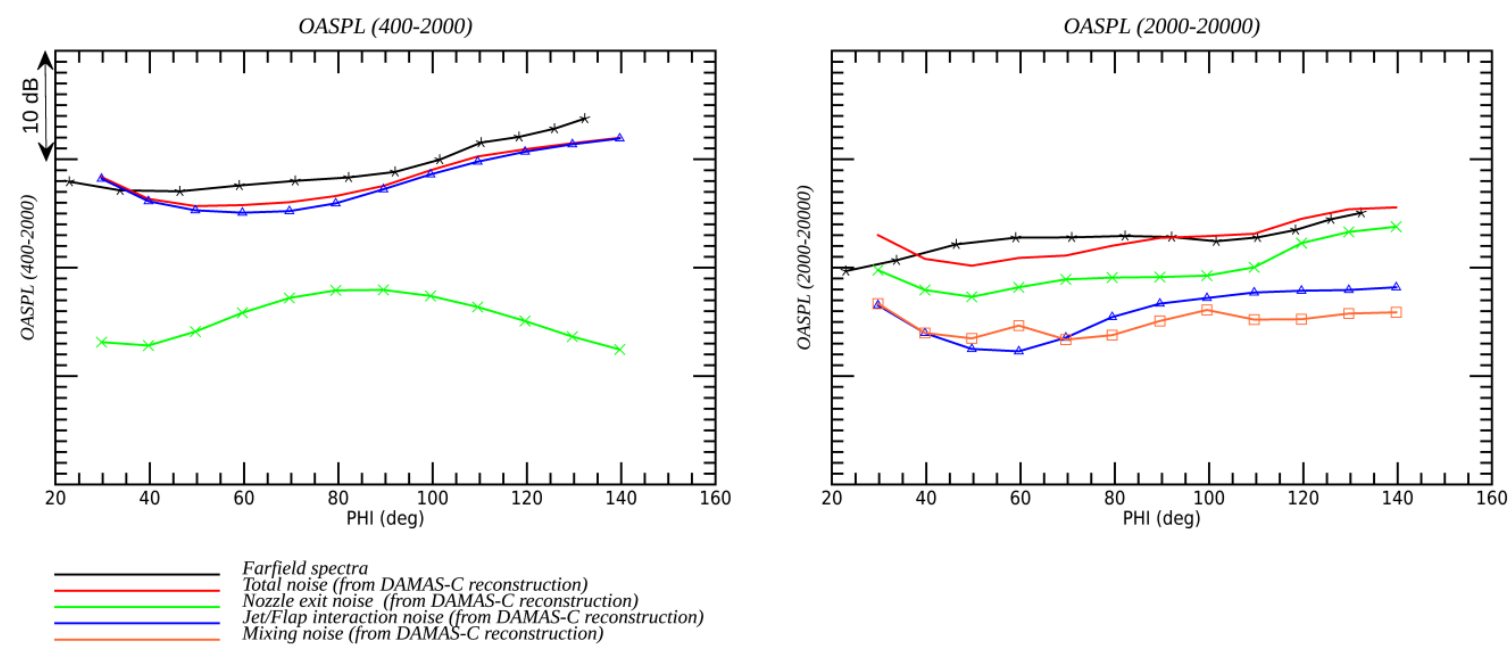

Fig. 14: Comparison of direct far field measurements with reconstructed source spectra from DAMAS-C sources identification. Installed nozzle: OP10- Flight effect $(90 \mathrm{~m} / \mathrm{s})$

\section{Installed jet-Flap tonal noise}

The effect of ever closer jet/wing installation has been investigated, as specific mechanism could appears for particular configuration as reported recently by different authors ${ }^{13,14}$. The spectra are dominated by strong tones as shown in Fig. 15 where the farfield spectra obtained for several jet-flap spacing are compared. The tones amplitudes increase as the jet is closer to the flap.

For the closest installation, array processing using correlated monopole model was applied (see Fig. 16). For the first tone two sources positions are localized at bypass exhaust and at the flap trailing edge (about $0.6 \mathrm{~m}$ spacing). Furthermore on the right the sources cross spectral matrices are plotted at the tone frequency and for another frequency in broadband range. These results exhibit the different nature of the noise sources. In the broadband domain, we do not have strong correlation between both positions as the extradiagonal terms corresponding to sources cross-spectra present low level. In contrast at the tone frequency; strong correlation is obtained, suggesting a coupling phenomenon between these locations. 

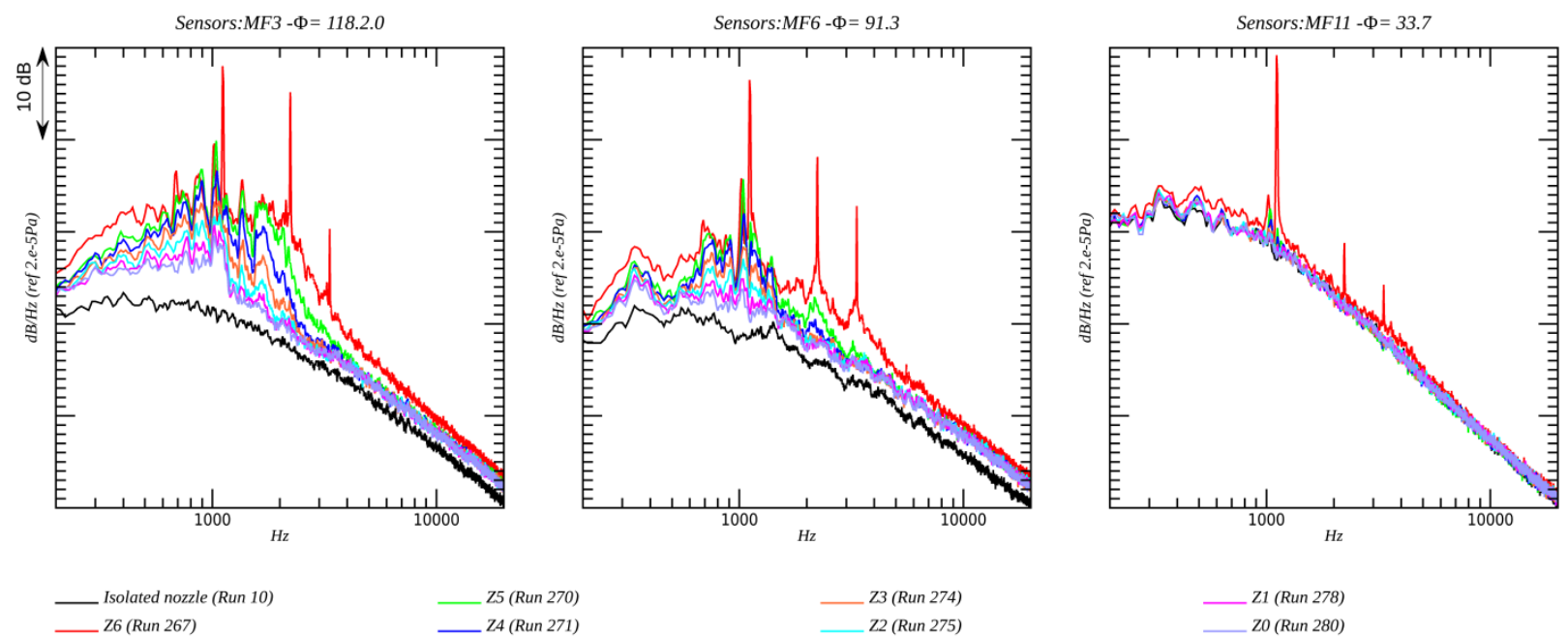

Z4 (Run 271)
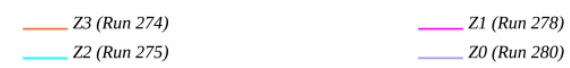

Fig. 15: Influence of the jet/flap distance (increasing from $\mathrm{Z6}$ to $\mathrm{ZO}$ ) on the far field spectra - OP3- Flight effect $(60 \mathrm{~m} / \mathrm{s})$

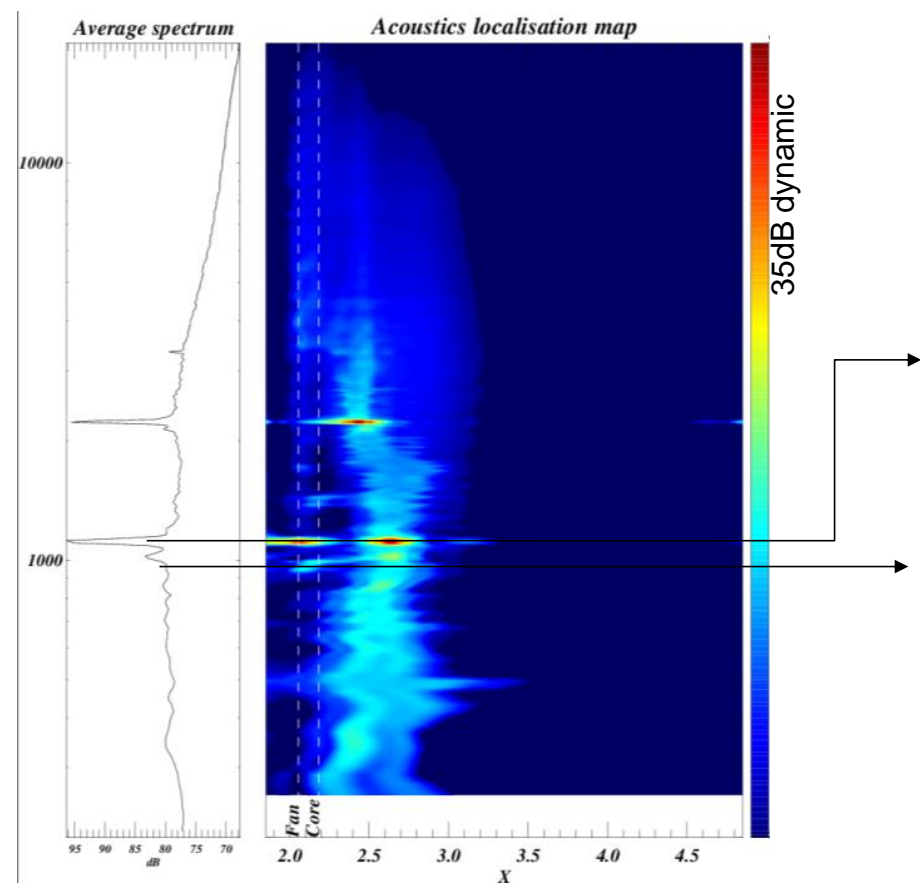

\section{Sources cross spectral matrices}
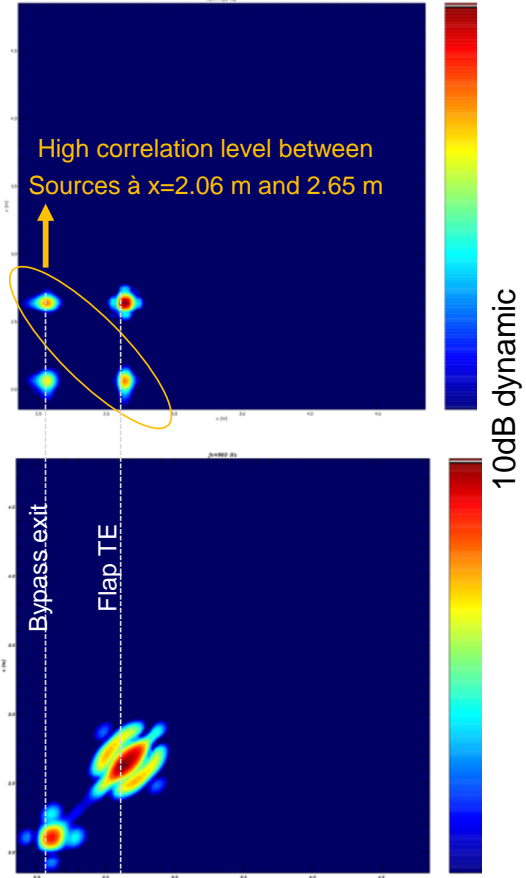

Fig. 16: Tonal jet/flap interaction noise identification from DAMAS-C -Installed x1-z6 - OP3- Flight effect $(60 \mathrm{~m} / \mathrm{s})$

\section{Conclusion}

The identification method developed at ONERA for jet noise has been applied here for the analysis of UHBR jet noise and the strong installation effect induced by the close-coupled configuration. Even if the uncorrelated monopoles model commonly used provides a valuable estimation of the sources positions, it has been demonstrated that for physics as jet noise and jet/flap interaction noise more complex sources model must be considered. For this reason the analysis has been done in considering correlated monopoles. The results of the sources cross spectra have been projected in farfield to assess these results by comparison with the direct farfield measurement. Fairly good agreement was obtained especially for installed configuration. Nevertheless for the quietest conditions with high flight effect, the mixing noise sources expected downstream near about 6 bypass diameters was not detected. The present investigation suggests that the dynamic of the method was not sufficient because we cumulate a significant internal rig noise associated with an antenna location which do not covered 
the main direction of jet noise radiation. The numerical simulations performed at ONERA were used to confirm this hypothesis, but also to evaluate their capacity to simulate such complex geometry. Acoustic array processing applied to these data presents favorable comparison with the experimental data. The jet/flap interaction noise is accurately localized and the noise directivity well reproduced. Thanks to the correlated model, the tonal jet-flap interaction noise was highlighted and pointed out strong correlation between source at bypass nozzle lip and flap trailing edge.

\section{Acknowledgments}

This work has been carried out in the framework of the European $7_{\text {th }}$ Framework Project JERONIMO, contract number ACP2-GA-2012-314692.

\section{References}

$\left.{ }^{1}\right]$ T.F. Brooks, W.M.Jr Humphreys. A Deconvolution Approach for the Mapping of Acoustic Sources (DAMAS) Determined from Phased Microphone Array. 10th AIAA/CEAS Aeroacoustics Conference, Manchester, Great Britain, n. 1, 10-12 May 2004. ISSN AIAA-2004-2954.

$\left.{ }^{2}\right]$ T.F. Brooks, W.M.Jr H Humphreys. Extension of DAMAS Phased Array Processing for Spatial Coherence Determination (DAMAS-C). 12th AIAA/CEAS Aeroacoustics Conference, CAmbridge, Masssachusetts, n. 2 , 8-10 May 2006. ISSN AIAA-2006-2654.

$\left[{ }^{3}\right]$ F. Gand et al, Jet noise of a UHBR nozzle using ZDES: external boundary layer thickness and installation effects, 23rd AIAA/CEAS Aeroacoustics Conference, 5-9 June 2017, Denver, Colorado

$\left.{ }^{4}\right]$ V. Fleury, J. Bulté, and R. Davy, Determination of acoustic directivity from microphone array measurements using correlated monopoles, 14th AIAA/CEAS Aeroacoustics Conference, 6-7 May 2008, Vancouver, British Columbia, Canada

$\left.{ }^{5}\right]$ V. Fleury, V.; R. Davy.; Analysis of jet-airfoil interaction noise sources by using a microphone array technique, Journal of Sound and Vibration, vol. 364, pp. 44-66 (2016)

$\left[{ }^{6}\right]$ G.Elias,Experimental techniques for source location, von Kármán Lecture Series, Aeroacoustic and Active Noise Control, September, 1997, pp.1-35.

[7] P.C.Hansen, Rank-Deficient and Discrete Ill-Posed Problems: Numerical Aspects of Linear Inversion, SIAM, Philadelphia, 1998

$\left[{ }^{8}\right]$ L. Cambier, S. Heib, and S. Plot, "The Onera elsA CFD software: input from research and feedback from industry," Mechanics and Industry, vol. 14, no. 3, pp. 159-174, 2013. doi: 10.1051/meca/2013056.

[9] S. Deck, "Recent Improvements of the Zonal Detached Eddy Simulation (ZDES) Formulation," Theoretical and Computational Fluid Dynamics, vol. 26, pp. 523-550, 2012. doi: 10.1007/s00162-011-0240-z.

$\left[{ }^{10}\right]$ J. E. Ffowcs Williams and D. L. Hawkings, "Sound generation by turbulence and surfaces in arbitrary motion," Philosophical Transactions for the Royal Society of London, vol. A264, pp. 321-342, 1969.

$\left.{ }^{11}\right]$ G. Rahier, M. Huet, and J. Prieur, "Additional terms for the use of Ffowcs Williams and Hawkings surface integrals inturbulent flows," Computers and Fluids, vol. 120, pp. 158-172, 2015.

$\left.{ }^{12}\right]$ S. Redonnet and G. Cunha, "An advanced hybrid method for the acoustic prediction," Advances in Engineering Software,vol. 88, no. 0, pp. 30-52, 2015. doi: 10.1016/j.advengsoft.2015.05.006.

$\left[{ }^{13}\right]$ J. L. T. Lawrence and R. H. Self, "Installed Jet-Flap Impingement Tonal Noise", 21st AIAA/CEAS Aeroacoustics Conference, 22-26 June 2015, Dallas, TX

$\left[{ }^{14}\right] \mathrm{V}$. Jaunet et al., "Tonal dynamics and sound in free and installed turbulent jets",22nd AIAA/CEAS Aeroacoustics Conference, 30 May - 1 June, 2016, Lyon, France 